\title{
Rare treatable genetic diseases: Considering disorders with specific treatment based on molecular pathology
}

\author{
Romana Vulturar ${ }^{1,2}$, Laura Damian ${ }^{3,4}$, Cecilia Lazea ${ }^{5,6}$ \\ ${ }^{1}$ Department of Molecular Sciences, "Iuliu Hatieganu" University of Medicine and Pharmacy, \\ Cluj-Napoca, Romania \\ ${ }^{2}$ Cognitive Neuroscience Laboratory, "Babes-Bolyai“ University, Cluj-Napoca, Romania \\ ${ }^{3}$ Department of Rheumatology, Emergency Clinical County Hospital Cluj, \\ Centre for Rare Autoimmune and Autoinflammatory Diseases (ERN-ReCONNET), Cluj-Napoca, Romania \\ ${ }^{4} \mathrm{CMI}$ Reumatologie Dr. Damian, Cluj-Napoca, Romania \\ ${ }^{5}$ Department Mother and Child, Discipline Pediatrics I, \\ "Iuliu Hatieganu" University of Medicine and Pharmacy, Cluj-Napoca, Romania \\ ${ }^{6}$ Pediatrics I Clinic, Emergency Children Hospital, Cluj-Napoca, Romania
}

\section{INTRODUCTION}

More than 4,300 genes have been associated to date with recognizable monogenic genetic disorders, listed in the Online Inheritance in Man (OMIM), meaning almost 7,000 phenotypes with known molecular basis (1). Towards the effort to identify and treat inasmuch as possible a genetic disorder, the aim to personalize medicine for the patients with rare diseases and to improve outcomes avoiding or alleviating disability has become a priority in some systems (NHS, National Health Service in UK (2).

A disease is considered rare if it affects less than 5 people in a population of 10,000 individuals. In the United States, the Rare Diseases Act of 2002 defines a rare disease $(\mathrm{RD})$ strictly according to prevalence, specifically "any disease or condition that affects less than 200,000 people in the United States", or approximately 1 in 1500 people (3). Although the first clinical signs of Inborn Errors of Metabolism (IEM) usually present in infancy, there is an unknown proportion of cases that can appear in adolescence or adulthood (4). The number of rare diseases is estimated at $6,000-8,000$, over $80 \%$ being genetic; most of them have certain common characteristics and although the entity is rare, rare diseas- es as a whole are common, affecting $6-8 \%$ of the European population, or approximately 36 million Europeans are or will be affected by one $\mathrm{RD}(2,5)$. One in 17 people is affected by a rare disease in a certain moment (6). Thus, the estimated figure for rare diseases patients in Romania is 1.3 million. The European Commission has adopted a Strategy of the Rights of Persons with Disabilities 2021-2030, as part of the European Union's commitment to promoting, protecting and ensuring the full enjoyment of human rights by individuals with disabilities and complex needs. An earlier, faster and more accurate diagnosis of rare diseases across Europe is amongst the 8 recommendations enclosed in the Rare 2030 Foresight Study initiated by the European Parliament and co-funded by European Commission Pilot Project and Preparatory Actions Programme, which set out the roadmap for the next decade in the field of rare diseases $(7,8)$.

Romania has a National Plan for Rare Diseases integrated in the National Strategy of Public Health and considerable efforts have been made to set the stage for improving genetic testing by creating the national genetic network covering the whole country (9). There are 27 expertise centers in Romania, and some of them are integrated in European Net- 
works of Rare diseases. The National Alliance for Rare Diseases Romania (ANBRaRo) currently enrolls 48 patients' organization and there is a good collaboration between patients' organizations and specialists (5). That is why increased awareness regarding the potentially treatable rare genetic diseases - some of them with simple means - could lead to early referral to genetic testing and hopefully to search for these diseases, allowing timely intervention.

The neonatal screening in Romania is performed for only two diseases, and the personalized nutrition plan are scarce. A rare disease impacts greatly the family's life and has serious social impact. The lack of adequate and personalized therapies leads to permanent disabilities and adult patients have reduced chances to integrate on the market and to self-sustain. Also, in rare genetic disorders a timely diagnosis may help avoid the recurrence of other cases in the family.

\section{THE THERAPIES INVOLVED}

Dietary management is important in almost 100 diseases, there are nutritional, vitamin and trace elements, pharmacological, and even stem cell approaches and other novel promising therapeutic avenues have been opened. The majority is safe and effective, for instance, avoiding phenylalanine from the earliest days of life in phenylketonuria, or limiting galactose intake is effective in diseases affecting galactose metabolism - GALT, GALE, GALK1 and GALM mutations $(10,11)$. Supplements are used for biotinidase deficiency, for creatine deficiency syndromes, for instance, or synthetic medium-chain tri-glycerides are recently introduced as a source of calories and fatty acids for the treatment of pediatric and adult patients with molecularly confirmed long-chain fatty acid oxidation disorders (LC-FAOD) (12).

The majority of inborn errors of metabolism affect the nervous system; regarding the intellectual disability, in 2021 is registered an increase in number of treatable disorders - 116 (30\% increase) and exome sequencing has brought new insights into disorders and treatments, opening the perspectives to new trial methods (communication Clara Van Karnebeek May 2021, Course Neurometabolic diseases: "it’s all in the brain"). For urea cycle disorders there are recommendations for nitrogen scavenger agents and supplementation with L-arginine and/or L-citrulline to promote an alternative pathway of metabolism (13). The ketogenic diet has been successfully introduced to treat conditions with im- paired bioenergetics, which through incompletely defined mechanisms result in a wide spectrum of neurological symptoms (developmental delay, epileptic seizures, movement disorders) like glucose transporter type 1 deficiency, succinic semialdehyde dehydrogenase deficiency, pyruvate dehydrogenase complex deficiency, nonketotic hyperglycinemia, and mitochondrial cytopathies to varying degrees (14).

Medications, vaccination and blood products (immunoglobulins in several immune deficiencies) may be employed in some instances. Medical procedures (phlebotomia in hemochromatosis, mastectomy and ovariectomy in BRCA pathogenic variants, thyroidectomy in MEN2 - multiple endocrine neoplasia type 2 etc.) are therapies in certain genetic diseases.

In other rare monogenic autoinflammatory disorders, therapies addressing hyperinflammation make a difference (for instance anti-IL-1 medication such as anakinra or canakinumab in cryopyrinopathies, mevalonate kinase deficiency and others) (15).

There are several extremely valuable internet resources on this topic (e.g. Rx - genes.com, containing 633 diseases resulting from variants in 619 genes) (11). Of these, 19 genes refer to adult-onset diseases (APOB, APOE, BRCA1, BRCA2, BMPR1A, CP, EPCAM, HFE, LDLR, LDLRAP1, MLH1, MSH2, MSH6, MUTYH, PCSK9, PMS2, SLC30A2, SMAD4, UROD).

We outline the importance to acknowledge and focus on a rare disease which, once recognized, could benefit from a certain medical intervention.

The presentation of disorders from a pathogenic and clinical perspective could be useful, also presenting it in a nutshell for the busy clinician. Some important topics are the diseases treated with supplements or special diets, such as AGAT and GAMT deficiencies, the biotin deficiencies related syndromes or the GLUT1 deficiency and many others.

Because even clinicians have difficulties interpreting genetic tests reports, the use of simple terms, translatable for the non-geneticist doctor, would improve the disease understanding and the communication with the patient (16). We also think that the geneticist is a very important piece in the team centered on the patient in the verge of personalized medicine, and therefore highlight once more the collaboration between medical specialties in this respect.

\section{Acknowledgment}

All authors have equal contribution. 


\section{REFERENCES}

1. https://omim.org/statistics/geneMap.

2. NHS England Medical Directorate, Graham E. Improving outcomes through personalized medicine. 2016. Available at: https://www. england.nhs.uk/wp-content/uploads/2016/09/improvingoutcomespersonalize-medicine.pdf.

3. Julkowska D, Austin CP, Cutillo CM, Gancberg D, Hager C, et al. The importance of international collaboration for rare diseases research: a European perspective. Gene Ther. 2017; 24(9):562-571.

4. Sedel F, Lyon-Caen O, Saudubray JM. Therapy insight: inborn errors of metabolism in adult neurology - a clinical approach focused on treatable diseases. Nat Clin Pract Neurol. 2007;3(5):279-90.

5. Dorica Dan. Rare diseases in Romania, from the perspective of patients' associations. 22 dec 2020. MedicHub.

6. De Vrueh R, Baekelandt ERF, de Haan JMH: Priority Medicines for Europe and the World "A Public Health approach to Innovation", Update on 2004 Background Paper 6.19 Rare Diseases, 12 March 2013. Available at: https://www.who.int/medicines/areas/priority_ medicines/BP6_18Rare.pdf.

7. Eurordis: Strategy for the rights of persons with disabilities: Rare 2030 multi-stakeholder recommendations on the future of rare diseases policy, 21 Mar 2021. Available at: https://www.eurordis.org.

8. Eurordis - Juggling care and daily life - The balancing actof the rare diseases community. A Rare Barometer survey. Available at: http:// download2.eurordis.org.s3.amazonaws.com/rbv/2017_05_09Social.
9. Grigore V. Integrarea Planului National de Boli Rare in strategia nationala de sanatate. Rev. Politici de Sanatate 26 feb 2021.

10. Jameson $E$, Remmington T. Dietary interventions for phenylketonuria. Cochrane Database Syst Rev. 2020;7(7):CD001304.

11. Bick D, Bick SL, Dimmock DP, Fowler TA, Caulfield MJ, Scott RH. An online compendium of treatable genetic disorders. Am J Med Genet. 2021; 187(1):48-54.

12. Shirley M. Triheptanoin: First Approval. Drugs. 2020;80(15):1595-1600.

13. Häberle J, Burlina A, Chakrapani A, Dixon M, Karall D, et al. Suggested guidelines for the diagnosis and management of urea cycle disorders: First revision. J Inherit Metab Dis. 2019;42(6):1192-1230.

14. Gavrilovici C, Rho JM. Metabolic epilepsies amenable to ketogenic therapies: Indications, contraindications, and underlying mechanisms. J Inherit Metab Dis. 2021;44(1):42-53.

15. Welzel T, Benseler SM, Kuemmerle-Deschner JB. Management of Monogenic IL-1 Mediated Autoinflammatory Diseases in Childhood. Front Immunol. 2021 Mar 18;12:516427.

16. Recchia G, Chiappi A, Chandratillake G, Raymond L, Freeman ALJ. Creating genetic reports that are understood by nonspecialists: a case study. Genet Med. 2020; 22(2):353-361.

Conflict of interest: none declared Financial support: none declared 